
\title{
CIÊNCIA'NATURA
}

\section{Descrição temporal do comportamento do Cerrado sensu strictu usando séries temporais}

\author{
Temporal description of Cerrado sensu stricto behavior using time series
}

Sérgio Luís Dias Machado, Claudionor Ribeiro Silva e Aracy Alves de Araújo

Universidade Federal de Uberlândia, Uberlândia, Minas Gerais, Brasil

\section{Resumo}

Atualmente tem ocorrido uma maior preocupação com a relação entre o ser humano e a natureza. Os desafios para que essa relação seja mais sustentável têm sido apontados. Juntamente com essa crescente necessidade e preocupação ambiental, as geotecnologias aparecem como forte aliadas, especialmente na geração de dados mais acurados e atualizados, para auxiliar em tarefas tais como o manejo e o planejamento ambiental em processos de exploração de um determinado bioma. Nesse contexto, esse estudo buscou analisar o comportamento fenológico de fitofisionomias do Cerrado (Estação Ecológica de Pirapitinga - MG) com o uso de séries temporais de NDVI e temperatura de superficie, determinados a partir de imagens digitais, obtidas com o sensor Landsat 5/TM. Para tanto, foi realizado um estudo com as duas séries temporais, analisando as variáveis intrínsecas, tais como tendência, sazonalidade, previsão e cointegração. A previsão da série NDVI apresentou um erro padrão inferior a 0,079, o que representa uma informação de qualidade acerca da fitofisionomia analisada em um tempo futuro. É um dado muito importante, tendo em vista que atualmente o Cerrado sofre com um acelerado processo de degradação. Portanto, é uma informação útil em processos de recuperação, manejo e gestão de áreas degradadas.

Palavras-chave: Estação Ecológica Pirapitinga. Temperatura de superficie. NDVI.

\section{Abstract}

Currently has been a growing concern about the relationship between humans and nature. The challenges to making this relationship more sustainable have been pointed out. Together with this growing need and concern environmental, geotechnologies appear as strong allies, especially in the generation of more accurate and updated data to assist in tasks such as environmental management and planning in the processes of exploration of a given biome. In this context, this study aimed to analyze the phenological behavior of Cerrado phytophysiognomies (Pirapitinga Ecological Station - MG) with the use of time series of NDVI and surface temperature determined from digital images, obtained with the Landsat 5-TM sensor. For this, a study was carried out with the two time series, analyzing the intrinsic variables, such as trend, seasonality, prediction and cointegration. The prediction of the NDVI series presented a standard error lower than 0.079, which represents a quality information about the phytophysiognomy analyzed at a future time. This is a very important fact, given that currently the Cerrado suffers from an accelerated process of degradation. Therefore, it is useful information in processes of recovery, maneuver and management of degraded areas.

Keywords: Pirapitinga ecological station. Surface temperature. NDVI. 


\section{Introdução}

A ocupação humana, nos diferentes pontos do planeta, sempre causou consequências ao meio ambiente. A urgência de preservação decorre da deterioração da natureza e do seu uso sem medidas e imprudente. Entretanto, a preocupação ambiental que antes quase não existia ou era pouco evidente, começou a ter destaque na sociedade, principalmente na comunidade científica. Com a evolução tanto do pensamento quanto da tecnologia, a forma de observar, julgar e tomar medidas referentes ao meio ambiente foram se modificando. Passou-se a observar os ambientes localmente, regional e globalmente, por entender que eles se correlacionam (JACOBI, 2005; SPAREMBERGUER; SILVA, 2005).

Devido às demandas atuais por diferentes estudos ambientais, o uso da tecnologia tornou-se essencial. Nesse contexto, diversos estudos usando as geotecnologias, como base para aquisição, processamento e análise de dados espaciais, bem como para auxiliar na tomada de decisões sobre variados temas, como aqueles relacionados à questão ambiental. O sensoriamento remoto por satélites é uma das ferramentas das geotecnologias útil nesse processo de coleta de dados espaciais (imagens digitais), com resolução espacial e temporal adequadas para estudos ambientais.

Esse estudo teve por objetivo analisar e predizer temporalmente o comportamento da fitofisionomia "Cerrado Sensu Strictu" do bioma Cerrado, usando duas séries temporais obtidas a partir imagens orbitais. As duas séries usadas foram de Índice de Vegetação da Diferença Normalizada (Normalized Difference Vegetation Index - NDVI) e de temperatura de superfície (TSUP), obtidas a partir de imagens Landsat 5/TM, no período de 1984 a 2010. Mais especificamente, essas duas séries temporais foram analisadas para descrever o comportamento temporal da cobertura vegetal da fitofisionomia Cerrado Sensu Strictu, por meio dos parâmetros intrínsecos da série temporal: tendência, sazonalidade, estacionariedade, previsão e cointegração. Essas informações são importantes para ações de prevenção, gestão e monitoramento do Cerrado, especialmente em estações ecológicas, como a de Pirapitinga, que foi analisada nesse estudo.

\section{Cerrado}

O Cerrado caracteriza-se como uma formação do tipo savana tropical, com destacada sazonalidade e presença, em diferentes porções, de formações herbáceas, arbustivas e arbóreas (EITEN, 1994; RIBEIRO; WALTER, 1998). A atividade agropecuária dentro do Cerrado tem sido bastante intensa, contando com culturas extensas de milho, soja, feijão e outros, o que tem causado um enorme prejuízo para o ecossistema, devido ao desmatamento das áreas e migração de animais. Segundo o Ministério do Meio Ambiente, o Cerrado é um dos hotspots da biodiversidade mundial, sendo a savana mais rica do mundo.

O Cerrado é um dos biomas terrestres de maior endemismo e diversidade de espécies (CASTRO, 1999; OLIVEIRAFILHO; RATTER, 2002). Abriga 11.627 espécies de plantas já catalogadas, 199 espécies de mamíferos, 837 espécies de aves, além da quantidade elevada de peixes, répteis e anfíbios. Somada a essa grande biodiversidade, o Cerrado ainda tem uma série de fitofisionomias com características particulares. Assim como a maioria das savanas do mundo, o Cerrado não é um tipo homogêneo de habitat, mas sim um mosaico de fisionomias vegetais que incluem desde campos abertos, savanas propriamente ditas, até florestas densas (OLIVEIRA-FILHO; RATTER, 2002).

Apesar dessa biodiversidade imensa e importante, segundo o Ministério do Meio ambiente, estima-se que $20 \%$ de todas as espécies endêmicas e nativas já não têm ocorrências em áreas protegidas e pelo menos 127 espécies animais desse habitat estão ameaçadas de extinção. Segundo Machado et al. (2004), apenas 20\% da cobertura original do Cerrado permanece intacta e somente $2,2 \%$ de sua área original de ocorrência está inserida em áreas protegidas. A retirada da cobertura vegetal e suas consequências totais são oriundas das atividades humanas que, com o passar dos anos, foram sendo desenvolvidas sem a preocupação ambiental necessária. Diante dessa situação em que o Cerrado se encontra, tornam-se necessários estudos que registrem informações para ações futuras, como de recuperação e monitoramento, bem como para gestão de áreas protegidas do bioma.

\section{NDVI e Temperatura de Superfície}

O sensoriamento remoto orbital permite imagear grandes áreas por um custo relativamente baixo, o que o destaca em relação a outras formas de obtenção de dados espaciais. Esses sensores captam as características espectrais de alvos como vegetação, solo e água, que são elementos fundamentais e essenciais para a análise e interpretação de dados em sensoriamento remoto (QUEIROZ, 1996). Cada alvo registrado pelo sensor se comporta de uma forma diferente, com valores digitais particulares, que variam de acordo com a faixa do espectro eletromagnético em que o sensor está configurado e com a forma com que os alvos reagem à radiação eletromagnética (NOVO, 2010; PONZONI; SHIMABUKURO, 2010; GIRI, 2016).

Essa característica espectral da imagem é importante porque permite analisar detalhes de um objeto, como uma espécie arbórea ou um conjunto delas, como uma fitofisionomia. Além dessa característica, merece ser destacada a resolução temporal das imagens, ou seja, o tempo que o sensor leva para margear uma mema área novamente, que permite uma análise temporal de um determinado objeto ou fenômeno, como a vegetação. Quanto menor for esse tempo de revisita, maior será a série temporal, podendo ser diária, quinzenal e/ou mensal.

Para a extração de alguma informação particular, como a vegetação, em uma imagem digital, é necessário usar alguma 
técnica específica de processamento digital de imagem que garanta o isolamento dessa informação em detrimento de outras. São técnicas como classificação, segmentação e ou cálculo de índices. Dentre essas técnicas estão os diversos índices de vegetação, criados a partir de operações aritméticas com imagens (bandas espectrais) (LIESENBERG, et al., 2007; CARVALHO et al., 2008; PONZONI; SHIMABUKURO, 2010).

Um dos índices mais conhecido para realçar as variações na densidade da cobertura vegetal é o índice de vegetação por diferença normalizada. Esse índice usa as bandas espectrais que são coletadas nos intervalos do espectro eletromagnético onde a vegetação possui maior reflectância (Infravermelho: 760 - 900nm) e maior absorção da radiação (vermelho: 630 - 690nm). O cálculo é feito pela razão da subtração pela soma dessas duas bandas (RAFIQUE et al, 2016; XU et al., 2016; YUAN, 2015). Segundo Meneses (2012), o NDVI possui a vantagem sobre os demais índices de vegetação por ser mais proporcional à biomassa da vegetação e sofrer menor influência das condições atmosféricas, o que o qualifica como preferível para fazer comparações ao longo do tempo, como é a proposta desse trabalho.

Outro índice interessante determinado a partir de imagens orbitais, é a temperatura de superfície. As imagens utilizadas para esse fim são coletadas na faixa espectral da radiação termal $(8,0-14,0 \mathrm{~nm})$, que é a faixa espectral onde o sensor capta a energia emitida pelos alvos (STEINKE et al., 2010). O índice é determinado pela conversão dos valores da radiação emitida pelos alvos em valores de temperatura. Malaret et al. (1985) propôs um modelo matemático que realização essa conversão.

\section{Série Temporal}

Para compreender como o ambiente se comporta com o passar dos anos, estudiosos utilizam-se de observações de fenômenos e objetos, para assim tentar compreender melhor o ambiente e suas particularidades. Essas observações são chamadas de séries temporais, que é uma coleção de variáveis aleatórias ordenadas no tempo, isto é, são coletadas por um processo estocástico (WOOLDRIDGE, 2002; COELHO JUNIOR et al., 2006; GUJARATI, 2011).

A partir da análise de como os fenômenos ocorrem no tempo, pode-se então fazer previsões de como esses eventos voltarão a acontecer no futuro, porém, para isso, é necessário que o processo observado seja estacionário. Gujarati (2011) define que um processo estocástico será chamado estacionário se a média e a variância forem constantes ao longo do tempo e o valor de covariância entre os dois períodos de tempo depender apenas da distância, do intervalo ou da defasagem entre os dois períodos e não do tempo real ao qual a covariância é computada.

A metodologia Box-Jenkins tem como objetivo, segundo Pokorny (1986), de identificar e estimar modelos estatísticos que podem ser interpretados como tendo sido gerados pelos dados amostrais em questão, onde deve-se admitir que suas características são constantes ao longo do tempo, em períodos passados, presentes e futuros. Essa metodologia considera o número de vezes que a série precisa ser diferenciada para ser estacionária, representado por $d$, os números de termos autorregressivos representado por $p$ e o número de termos de média móvel representador por $q$.

Gujarati (2011) apresenta que a metodologia Box-Jenkins precisa seguir quatro diferentes passos: inicia-se a modelagem pela etapa 1, que consiste na identificação dos valores apropriados de $p, d$ e $q$; segue-se então na etapa 2, que é a estimação de parâmetros dos termos autorregressivos e de média móvel do modelo; a etapa 3 consiste na verificação do diagnóstico, que é determinar se o modelo ARIMA selecionado ajusta-se de forma razoável a outro modelo ARIMA; por fim, tem-se a etapa 4, que é a previsão propriamente dita.

\section{Material e Método}

A Estação Ecológica de Pirapitinga (EEP) foi criada em 20 de julho de 1987, pelo Decreto 94.656 e conta com uma área de aproximadamente 1.090ha, segundo seu Plano de Manejo (INSTITUTO CHICO MENDES, 2013). A Estação Ecológica fica numa ilha artificial no reservatório da Usina Hidrelétrica de Três Marias, na região do Alto Rio São Francisco (Figura 1). O clima da região é definido por Köppen como Aw, ou seja, um clima quente e úmido, com estação seca de inverno. A vegetação é predominantemente de Cerrado. Está localizada no município de Morada Nova de Minas, geograficamente entre as coordenadas $18^{\circ} 20^{\prime} \mathrm{S}-18^{\circ} 23^{\prime} \mathrm{S}$ e $45^{\circ} 17^{\prime} \mathrm{W}-45^{\circ} 20^{\prime} \mathrm{W}$, com altitudes que variam entre $560 \mathrm{~m}$ até $630 \mathrm{~m}$.

Nesse estudo foram utilizadas imagens orbitais do sensor TM, acoplado no satélite Landsat 5, referentes aos anos de 1984 até 2010. Foram utilizadas as bandas 2 (0,52 a 0,60 $\mu \mathrm{m}$ - verde), $3(0,63$ a 0,69 $\mu \mathrm{m}$ - vermelho), 4 ( 0,76 a $0,90 \mu \mathrm{m}$ - infravermelho próximo) e a 6 (10,4 a 12,5 $\mu \mathrm{m}$ - infravermelho termal). A resolução espacial das três primeiras bandas é de 30 metros e da banda 6 (termal) de 120 metros. Entende-se que essa diferença de resolução não interfere nos resultados, uma vez que a banda 6 é usada para calcular a temperatura de superfície da Terra, o que não altera consideravelmente dentro da área de 120 metros quadrados, podendo ser reamostrada para 30 metros sem perder qualidade na informação. Todas as imagens são disponibilizadas gratuitamente pelo site oficial do Instituto Nacional de Pesquisas Espaciais (INPE).

Os procedimentos de recorte, registro e elaboração dos mapas temáticos foram feitos com uso do software QGIS 2.18.3, disponibilizado gratuitamente. Os cálculos dos valores de NDVI e temperatura de superfície foram feitos utilizando a função "band math", implementada no software ENVI 5.0, pertencente ao laboratório SIGEO/UFU. Por fim, o processamento das 


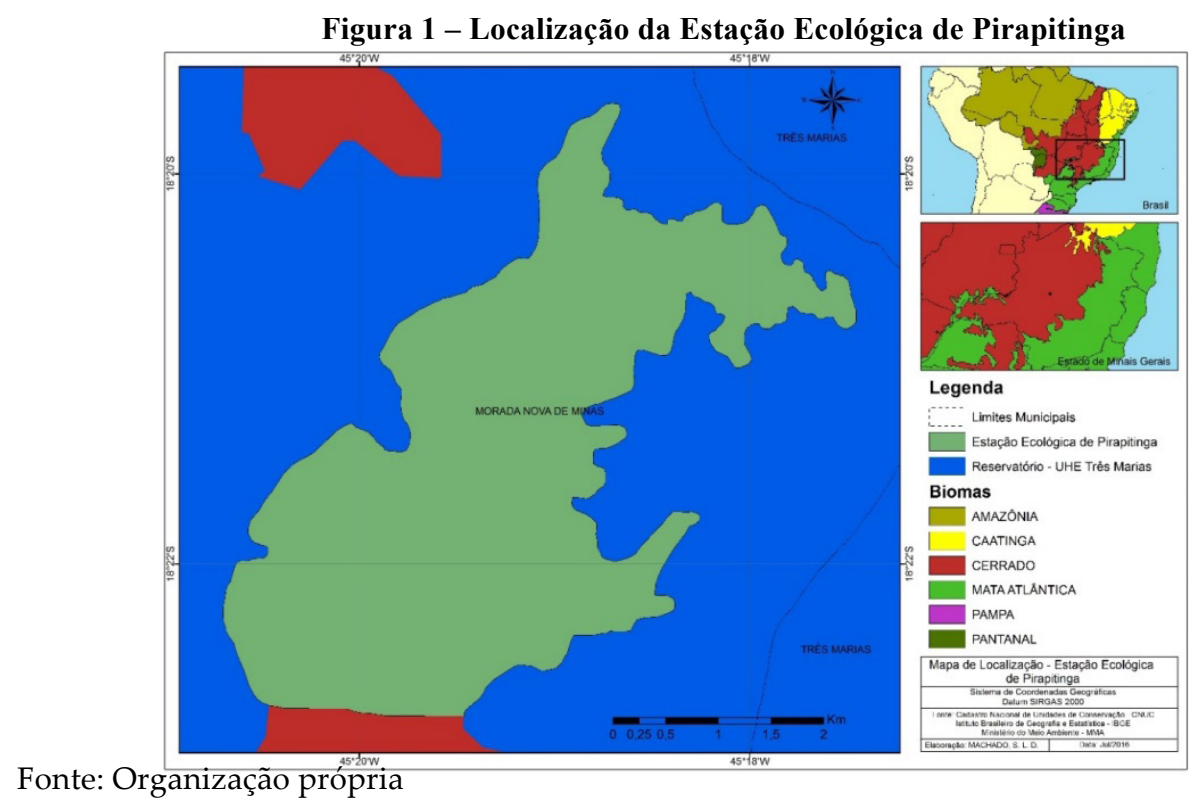

séries temporais foi realizado com o software Gretl, que também é um software livre.

Para preparação das imagens, inicialmente foi realizado um recorte retangular, em todas as cenas, que envolve a área de estudo. Em seguida, todas as cenas foram registradas, tendo por base a imagem mais antiga, do ano de 1984. Nesse processo, foram geradas as composições em falsa-cor (R,G,B: 3,4,2), para facilitar a localização/visualização dos pontos de controle. Após o registro, foram criados dois arquivos: um contendo a série com as bandas 3 (vermelho: R) e 4 (infravermelho: IR), para cálculo do NDVI, e outro contendo a série com as bandas termais, para cálculo da temperatura de superfície.

O cálculo do NDVI foi realizado por meio da Equação 1, proposta por Rouse et al (1973).

$$
N D V I=\frac{I R-R}{I R+R}
$$

A temperatura de superfície foi determinada com base no modelo (Equação 2) proposto por Malaret et al. (1985). Esse modelo foi adaptado para obtenção direta da temperatura de superfície em graus Celsius, isto é, subtraído de $-273,15$.

$$
T\left({ }^{\circ} \mathrm{C}\right)=\left(0,831 D N-0,00133 D N^{2}\right)-63,319
$$

onde: é a temperatura de superfície em graus Celsius; e é o valor do número digital presente na imagem termal.

Em alguns meses a presença de nuvens impossibilitou a coleta de dados, e para solucionar o problema de ausência de dados foi usado o método de interpolação por krigagem. A krigagem é um método de interpolação de dados que faz uso de funções matemáticas que acrescentam pesos maiores em posições mais próximas as amostras e pesos menores para as posições que se encontram mais distantes dos pontos amostrados, gerando assim, novos pontos interpolados baseados em combinações lineares de dados (JAKOB, 2002; HAIR et al., 2005).

A aplicação da série temporal foi realizada em quatro fases: na primeira fase analisou-se visualmente os gráficos gerados a partir das séries, para observar e identificar a presença de outliers. Para verificar se as séries têm tendência, aplicou-se os testes de tendência "simples", "logarítmica" e "quadrática", utilizando o método dos Mínimos Quadrados Ordinários (MQO). Nesses testes, os p-Valores medidos na série indicam se o modelo está bem ajustado nos intervalos de confiança $1 \%, 5 \%$ e $10 \%$.

$\mathrm{Na}$ segunda fase foi verificado se as séries possuem sazonalidade, observando os periodogramas das séries, onde a sazonalidade é indicada por meio da ocorrência de picos individualizados. Em caso de necessidade, a série deve ser suavizada por meio da aplicação de diferenças sazonais, para posterior modelagem.

A análise da estacionariedade das séries foi executada na terceira fase, verificando a existência de raiz unitária, usando um modelo autoregressivo. No teste da raiz unitária, a hipótese nula é de que a série testada possui raiz unitária e, portanto, é não-estacionária. A hipótese alternativa é de que a série não possui raiz unitária e é estacionária. Para aceitar, o p-Valor deve ser maior que os níveis usuais de significância (1\%,5\% e 10\%). Um teste bastante utilizado para análise da raiz unitária é o ADF (Dickey-Fuller Aumentado), modelado pela Equação 3. Quando o teste aponta que a série é estacionária, deve-se aplicar defasagens na série e testa novamente com o ADF, verificando se a série defasada é não-estacionária (aceita ).

$$
\Delta y_{t}=\beta_{1}+\beta_{2} t+\delta y_{t-1}+\sum_{1}^{m} \alpha_{i} \Delta y_{t-1}+\varepsilon_{t}
$$


onde: é a variável de interesse; é o intercepto, também denominado de drift da série; é o coeficiente de uma tendência temporal; é o coeficiente de presença de raiz unitária; m é o número de defasagens tomadas na série; é o erro estocástico; e é o operador de defasagens.

Por fim, a previsão das séries foi realizada utilizando o método SARIMA, com modelo X-12-ARIMA (Máxima Verossimilhança Exata), significativo nos níveis usuais de significância (1\%, 5\% e 10\%). Nessa mesma fase foi aplicada a cointegração entre as duas séries, usando o método de Engle Granger, que consistiu na aplicação do teste ADF na série dos resíduos e na consequente verificação da existência de raiz unitária, para saber se essa série era estacionária.

\section{Resultados}

Toda a análise da série temporal foi realizada com a série NDVI, de maior importância para esse estudo. A série TSUP foi usada apenas para calcular a cointegração e verificar se há influência direta da temperatura de superfície nos valores de NDVI. Na Figura 2 são apresentados os gráficos das séries de tempo de NDVI (Figura 2a) e TSUP (Figura 2b), correspondentes à fitofisionomia "Cerrado Stricto Sensu".

Figura 2 - Séries de NDVI (a) e TSUP (b) referentes à fitofisionomia Stricto Sensu
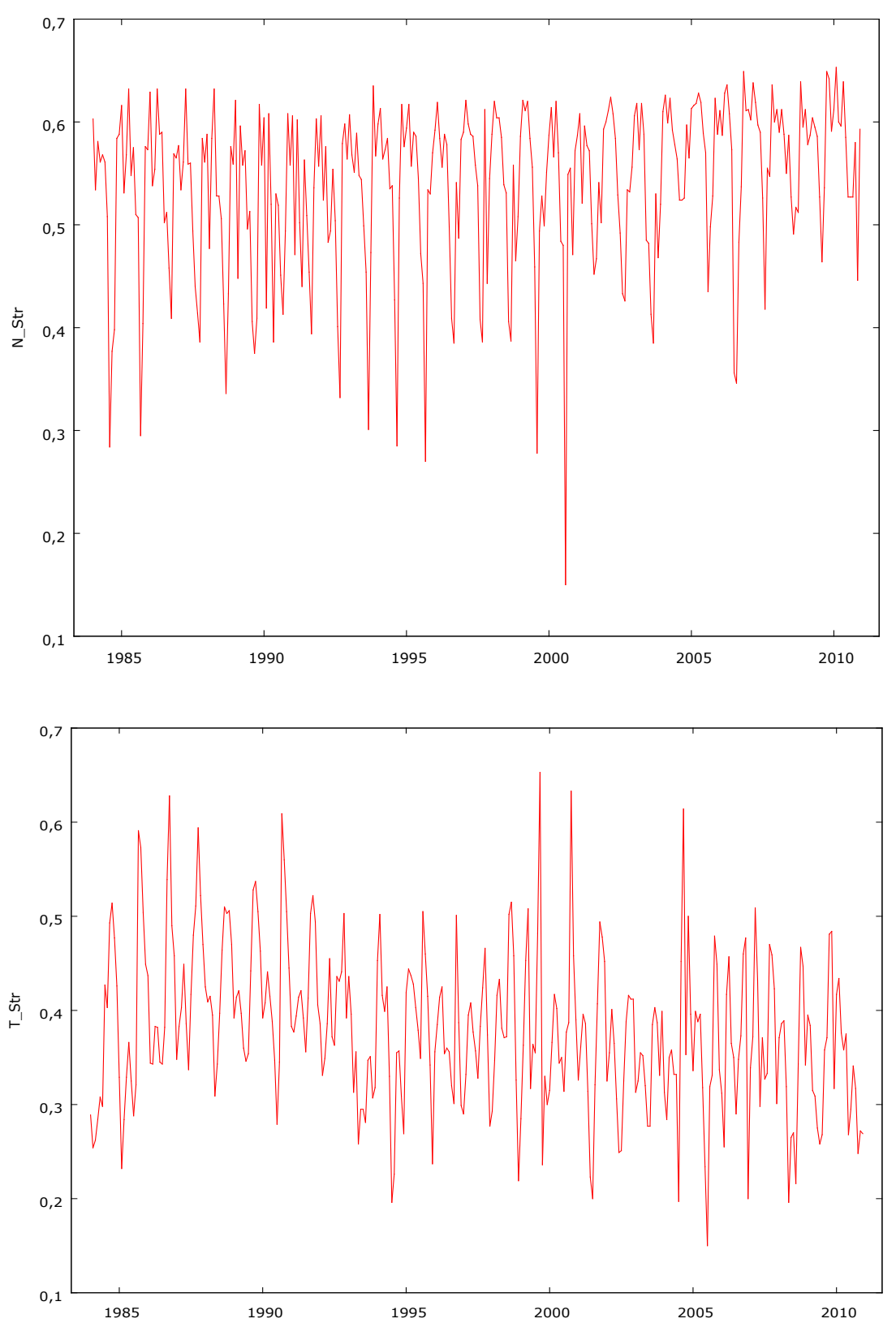


\subsection{Análise de Tendência, Sazonalidade e Estacionaridade}

A partir de uma análise visual na Figura 02, nota-se que não há a presença de outliers e não é claro se há presença de tendência, o que demanda a aplicação do teste de identificação de tendência na série NDVI.

Para verificar a tendência foram aplicados os três testes: simples, logarítmica e quadrática. O primeiro teste (tendência simples), realizado com a adição de uma variável de tempo (time), mostrou resultado positivo para a presença de tendência, sendo significativo a 1\%. Nesse teste foi utilizado o método dos Mínimos Quadrados Ordinários (MQO), com 324 observações, coletadas mensalmente no período de 01/1984 a 12/2010. Os resultados apontaram que há a presença de tendência no modelo com a variável (time/tendência), como mostrado na Tabela 1.

Tabela 1 - Resultados do teste de tendência simples, quadrática e logarítmica para NDVI

\begin{tabular}{c|c|c|c|c}
\hline & Coeficiente & Erro Padrão & Razão-t & p-Valor \\
\hline Constante & 0,510120 & 0,008717 & 58,520 & $1,190 \mathrm{e}-173 * * *$ \\
\hline Tendência Simples & $1,776 \mathrm{e}-04$ & $4,649 \mathrm{e}-05$ & 3,820 & $2,000 \mathrm{e}-04 * * *$ \\
\hline Constante & 0,527219 & 0,013084 & 40,290 & $1,340 \mathrm{e}-127 * * *$ \\
\hline Tendência Quadrática & $9,68332 \mathrm{e}-07$ & $5,53999 \mathrm{e}-07$ & 1,748 & $0,081 *$ \\
\hline Constante & 0,821396 & 0,002898 & 283,400 & $0,000 * * *$ \\
\hline Tendência Logarítmica & 0,447097 & 0,004420 & 101,100 & $4,580 \mathrm{e}-246 * * *$ \\
\hline
\end{tabular}

*** indica que o resultado é significativo a 1\% e * significativo a 10\%. Fonte: Organização própria

A variável dependente apresentou o índice R-quadrado ajustado igual a 0,040387, 0,046472 e 0,969390, respectivamente, para tendência simples, quadrática e logarítmica. Os p-valores resultantes foram, respectivamente: 0,000160 (para $\mathrm{F}=1,322$ ), 0,000178 (para $\mathrm{F}=2,321$ ) e 4,600e-246 (para $\mathrm{F}=1,322$ ). Os p-valores menores que 0,01 confirmam a existência de tendência na série NDVI, em um intervalo de confiança de $99 \%$.

A análise para identificar a presença do componente sazonal foi realizada no periodograma da série NDVI. Ocorreram picos individualizados no periodograma, mostrando que há um comportamento sazonal. Para modelar as sazonalidades na série NDVI foi aplicada uma diferença sazonal, tornando-a suavizada. Após a suavização das séries, com uma defasagem, iniciou-se a análise da estacionaridade.

A estacionariedade foi verificada pela análise da existência de raiz unitária na série NDVI. Ao aplicar o teste ADF (Aumentado de Dickey-Fuller), observou-se que a hipótese nula (Ho) foi aceita, mostrando que a série NDVI possui raiz unitária, sendo, portanto, não-estacionária. Isso ocorreu porque a série apresentou um p-valor assintótico igual a 0,2475 , maior que os níveis de significância usuais. Para corrigir o problema, foi realizada uma defasagem (322 observações) e aplicado o teste ADF novamente. Após a defasagem, verificou-se que a série se tornou estacionária, devido ao baixo p-valor assintótico, igual a 4,644e-036, nesse caso, menor que os níveis de significância usuais.

\subsection{Aplicação do Modelo SARIMA para Previsão da Série}

Nessa fase, com a série já estacionária, foi possível aplicar o modelo SARIMA, para estimativa da previsão dessa série NDVI. Tendo como variável dependente o NDVI da fitofisionomia Cerrado stricto sensu e aplicando o modelo X-12-ARIMA (Máxima verossimilhança exata), com as 324 observações (01/1984 a 12/2010), foram determinados os seguintes coeficientes (Tabela 2).

Tabela 2 - Ajuste do modelo X-12-ARIMA com a série N_Str

\begin{tabular}{ccccc}
\hline & Coeficiente & Erro Padrão & $\mathrm{z}$ & $\mathrm{p}$-Valor \\
Constante & 0,000174 & $7,320 \mathrm{e}-05$ & 2,387 & 0,017 ** \\
$\pi 1$ & 0,430250 & 0,051331 & 8,382 & $5,210 \mathrm{e}-017 * * *$ \\
$\theta 1$ & $-0,999982$ & 0,016633 & $-60,120$ & $0,000 * * *$ \\
\hline
\end{tabular}

*** indica que o resultado é significativo a 1\% e ** significativo a 5\%. Fonte: Organização própria

Embora tenha apresentado uma "constante" significativa a 5\%, os baixos p-valores mostram que o modelo X-12-ARIMA é uma escolha acertada para o estudo do comportamento da série NDVI. Definido o modelo, foi possível realizar a previsão da série NDVI, para o ano de 2010, e comparar com a observação de NDVI do mesmo ano. Como estatísticas de avaliação dessa previsão são apresentados os erros resultantes: erro médio $(0,0045271)$, erro quadrático médio $(0,0030127)$ e o erro absoluto médio $(0,043484)$.

Dados os baixos valores dos erros na previsão com o modelo resultante do "X-12-ARIMA" foram estimados os valores de previsão da série de NDVI, medidos na fitofisionomia Cerrado stricto sensu, para o ano de 2011 (além da série original), em 
um intervalo de confiança $95 \%(z[0,025]=1,96)$. Os baixos valores de erro padrão $(<0,079)$ apontam uma previsão acertada, como ilustrado no gráfico da Figura 3.

Figura 3 - Previsão da série N_Str para o ano 2011

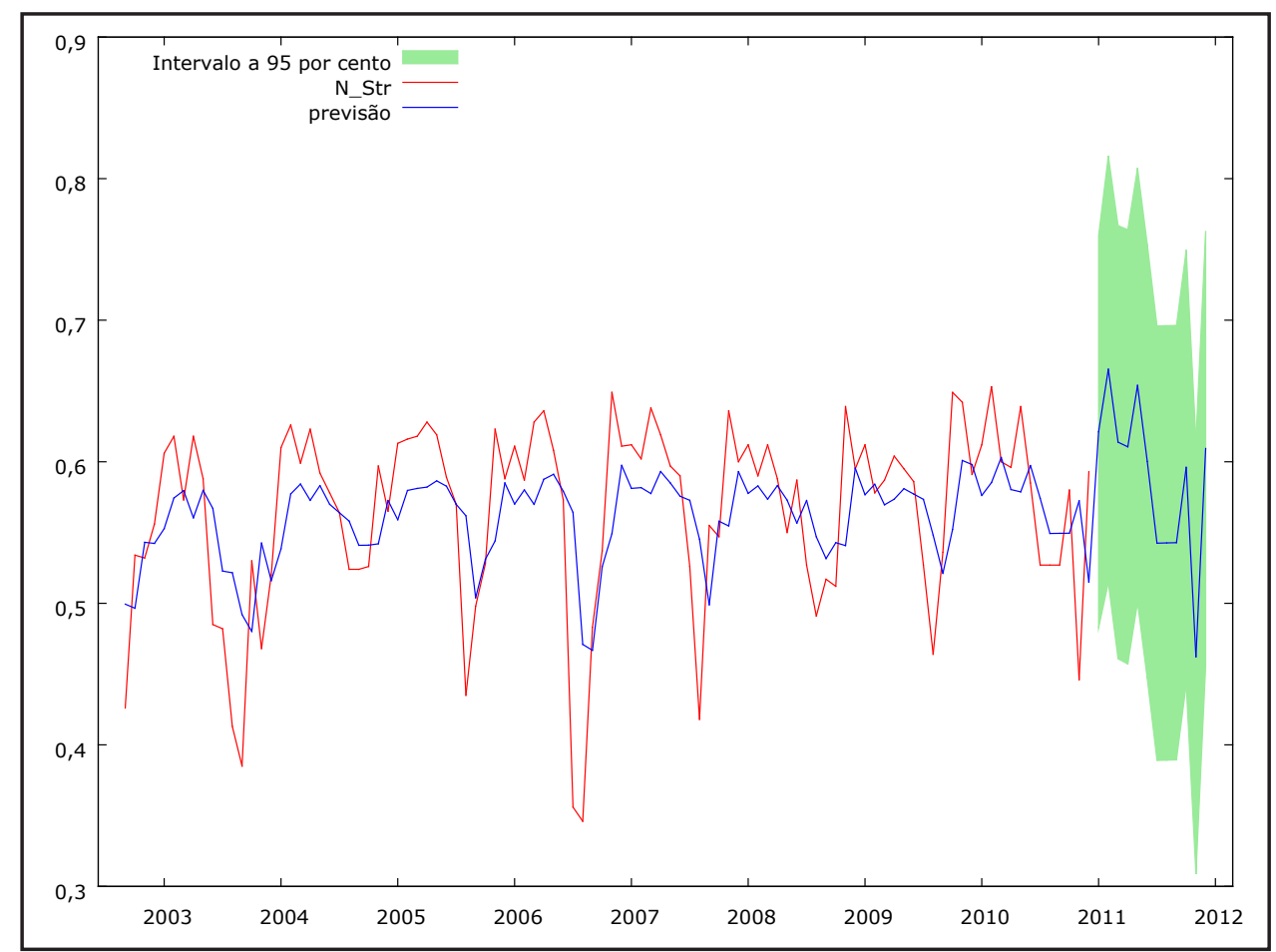

Fonte: Organização própria

\subsection{Cointegração das séries NDVI x TSUP}

O teste de cointegração entre as séries NDVI (dependente) e TSUP (independente) foi realizado pelo método de Engle Granger. Como ocorrido com a série original de NDVI, que apresentou raiz unitária, com p-valor igual a 0,2475, a série TSUP também apresentou raiz unitária, com p-valor igual a 0,1812. Contudo, após uma defasagem e nova aplicação do teste ADF, comprovou-se que as duas séries (NDVI e TSUP) se tornaram estacionárias (p-valores iguais a 4,644e-036 e 3,735e-033, respectivamente), condição necessária para aplicação do teste de cointegração. O baixo valor de $\mathrm{R}^{2}$ Ajustado $(0,023891)$ demonstra que a temperatura de superfície não tem um grande grau de explicação sobre a variação do NDVI.

A regressão de cointegração foi realizada pelo método MQO, usando as 324 observações coletadas no período (1984/01 a 2010/12), tendo como variável dependente a série TSUP. Dentro desse processo, no teste da raiz unitária dos resíduos, foi utilizado o teste ADF, encontrando um p-valor igual a 2,125e-020, o que significa que a série dos resíduos é estacionária.

Uma relação de cointegração entre duas variáveis/séries ocorre se e somente se: a) a hipótese de raiz unitária não é rejeitada para as variáveis individuais e b) a hipótese de raiz unitária é rejeitada para os resíduos da regressão de cointegração. No caso apresentado aqui, o critério (a) não é atendido, uma vez que a hipótese de raiz unitária é rejeitada, indicando que não há cointegração entre as duas variáveis: NDVI e TSUP.

\section{Discussões}

A operação do satélite Landsat 5/TM foi iniciada no ano de 1984 e interrompida no início do ano de 2011, o que permitiu a geração de uma série temporal de imagens de 26 anos, retirando o ano de 2011 (cobertura por nuvens), que eram basicamente os meses de janeiro e fevereiro. As duas séries temporais NDVI e TSUP apresentaram valores coerentes com a realidade da feição analisada, sendo ainda considerados dados de alta resolução espacial, se comparado com as séries obtidas a partir dos sensores AVHRR, MODIS e METEOSAT (NASSUR et al 2015; YUAN et al 2015; XU et al 2016).

Os resultados apontaram que há a presença de tendência no modelo para a série NDVI. Isso implica que essa variável pode ser descrita por um modelo de tendência simples, com resultados dentro de um intervalo de confiança de $1 \%$.

A presença da componente sazonal na série temporal NDVI era esperada, dado que a intensidade da clorofila e de folhagem 
das espécies arbóreas do Cerrado costuma variar com as mudanças das estações do ano, repetindo a cada ano. A análise do periodograma da série NDVI deixou claro essa premissa, ao apontar picos individualizados, o que representa a sazonalidade. A desconsideração desse parâmetro pode provocar prejuízos nos resultados, como apontado por Nassur et al 2015.

A série original NDVI mostrou ser não estacionária. De acordo com Diniz et al (1998), séries temporais sazonais ou com tendência linear ou exponencial são exemplos de séries temporais com comportamento não estacionário, o que acorda com o resultado encontrado nesse estudo. Contudo, a maioria dos procedimentos de análise estatística de séries temporais supõe que as séries sejam estacionárias, caso contrário, os dados originais devem ser transformados. Nesse estudo, a série NDVI foi submetida a uma defasagem, que foi o bastante para torná-la estacionária.

A previsão feita para o ano de 2010 mostrou como é possível descrever a variável NDVI no futuro, por pelo menos um ano, pois apresentou erro baixo. Esse resultado confiável de 2010 permitiu determinar a previsão para o ano de 2011 , período sem dados originais, em um intervalo de confiança de 1\%. Ressalta-se que a previsão obtida nesse estudo corresponde a um período de um ano, período relativamente longo, quando comparado com outro estudo, que restringiram a previsão em poucos dias (ALHAMAD et al 2007; NASSUR et al 2015).

Esperava-se que as séries NDVI e TSUP fossem cointegradas, uma vez que a temperatura teoricamente afetaria a intensidade de clorofila (maturidade) nas folhas de qualquer espécie arbórea. Contudo, os resultados mostraram o contrário, que não há cointegração. Acredita-se que esse fato se deve à característica da temperatura utilizada, correspondente à superfície da Terra, e não ao ambiente/espaço onde as folhas se encontram. A cointegração seria interessante uma vez que o cálculo do NDVI demanda dados muito específicos (bandas espectrais refletivas: vermelho e infravermelho), que são mais difíceis de serem encontrados com boa qualidade, especialmente devido à cobertura por nuvens.

\section{Conclusões}

As geotecnologias estão disponíveis para uso na ciência, possibilitando a obtenção de informações referentes à superfície terrestre, com qualidade e grande quantidade. As técnicas para elaboração das séries temporais e o uso de métodos estatísticos de séries temporais aplicados nesse estudo mostraram-se eficientes e confiáveis, com cálculo de previsão do NDVI de forma acertada, confirmada estatisticamente, sendo assim uma metodologia de grande potencial para auxiliar as demandas de preservação e planejamento ambiental.

O resultado/modelo encontrado pode ser utilizado em estudos relacionados às mudanças ocorridas no dossel da vegetação de áreas protegidas do Cerrado, especialmente para identificação de mudanças bruscas como incêndios, doenças e desmatamento em escalas local e regional.

Estudos futuros serão feitos inserindo novas variáveis, como a temperatura ambiente, ou novos métodos de cálculo da temperatura de superfície.

\section{Agradecimentos}

Os autores agradecem à Fundação de Amparo à Pesquisa do Estado de Minas Gerais - FAPEMIG pela concessão de apoio financeiro ao projeto, bem como apoio com bolsas de iniciação científica.

Os autores agradecem também ao Instituto Chico Mendes de Conservação da Biodiversidade (ICMBio) pelo apoio irrestrito à nossa equipe na Estação Ecológica de Pirapitinga, cedendo pessoal e equipamentos essenciais ao bom andamento da pesquisa.

\section{Referências}

ALHAMAD MN, STUTHT J, VANNUCCI M. Biophysical Modelling and NDVI Time Series to Project Near-Term Forage Supply: Spectral Analysis Aided by Wavelet Denoising and ARIMA Modelling. International Journal of Remote Sensing. 2007;28(11):2513-2548.

CARVALHO, FMV FERREIRA LG, LOBO FC, DINIZ-FILHO JAF, BINI, LM. Padrões de autocorrelação espacial de índices de vegetação MODIS no bioma cerrado. Revista Árvore, 2008;32(2):279-290.

CASTRO A, MARTINS FR, TAMASHIRO JY, SHEPHERD GJ. How rich is the flora of Brazilian Cerrados. Annals of the Missouri Botanical Garden. 1999;86(1):192-224.

COELHO JUNIOR LM, REZENDE JLP, CALEGARIO N, SILVA ML. Análise longitudinal dos preços do carvão vegetal, no Estado de Minas Gerais. Revista Árvore. 2006;30(3):429-438. 
DINIZ H, ANDRADE LCM, CARVALHO ACPLF, ANDRADE MG. Previsão de séries temporais utilizando redes neurais artificiais e modelos de box e jenkins. In: Anais V Simpósio Brasileiro de Redes Neurais [Internet]. 1998 December s/d; Belo Horizonte/MG, Brasil. 1998 [cited 2017 feb. 25]. Available from: http://repository.usp. br/.

EITEN G. Vegetação do Cerrado. In: PINTO M. N. (Ed.). Cerrado: caracterização, ocupação e perspectiva. Brasília, DF: Editora UNB; 1994.

GUJARATI DN, PORTER DC. Econometria Básica. 5 ed. Porto Alegre: Amgh Editora Ltd.; 2011.

INSTITUTO CHICO MENDES (ICMBIO). Estação Ecológica de Pirapitinga. Brasília (Brasil): Ministério do Meio Ambiente, 2013.

JACOBI PR. Educação Ambiental: o desafio da construção de um pensamento crítico, complexo e reflexivo. Educação e Pesquisa. 2005;31(2).

LAGO AAC. Estocolmo, Rio, Joanesburgo o Brasil e as três conferências ambientais das Nações Unidas. Brasília: Funag; 2006.

LIESENBERG V, PONZONI FJ, GALVÃO LS. Análise da dinâmica sazonal e separabilidade espectral de algumas fitofisionomias do cerrado com índices de vegetação dos sensores MODIS/TERRA e AQUA. Revista Árvore. 2007;31(2):295-305.

MENESES PR, ALMEIDA T, ROSA ANCS, SANO EE, SOUZA EB, BAPTISTA GMM, BRITES, RS. Introdução ao Processamento de Imagens de Sensoriamento Remoto. Brasília: Cnpq; 2012.

NASSUR OAC, FERREIRA E, SAFADI T, DANTAS, AAA. Monitoramento e Projeção Futura da Vegetação no Parque Nacional do Itatiaia Através de Sensoriamento Remoto. CERNE. 2015;21(3).

NOVO EMLM. Sensoriamento Remoto: Princípios e Aplicações. 4 ed. São Paulo: Blucer; 2010.

OLIVEIRA FILHO, A.T.; RATTER, J. T. Vegetation physiognomies and woody flora o the cerrado biome. In: Oliveira PS, Marquis RJ. (Ed). The cerrados of Brazil: ecology and natural history of a Neotropical savanna. New York: Columbia University Press; 2002. p. 91-120.

PINHEIRO ES, DURIGAN G. Geotecnologias aplicadas à análise da dinâmica do cerrado na Estação Ecológica de Assis, SP. In: Anais XIV Simpósio Brasileiro de Sensoriamento Remoto [Internet]; 2009 April 25-30; Natal, Brasil. 2009 [cited 2017 feb. 20]. Available from: http://marte.dpi.inpe.br/col/dpi.inpe.br/ sbsr@80/2008/11.09.14.30/doc/2905-2912.pdf.

POKORNY M. Introduction to Econometrics. Oxford: Basil Blackwell Ltd.;1987.

PONZONI FJ. SHIMABUKURO, Y. E. Sensoriamento Remoto no Estudo da Vegetação. São José dos Campos: Parêntese; 2010.

RAFIQUE R, ZHAO F, JONG R, ZENG N, ASRAR, GR. Global and Regional Variability and Change in Terrestrial Ecosystems Net Primary Production and NDVI: A Model-Data Comparison. Remote Sensing. 2016;8(3):177-193.

RIBEIRO JF, WALTER BMT. Fitofisionomia do Bioma Cerrado. In: Sano SM, Almeida SP. (Ed). Cerrado: ambiente e flora. Planaltina, DF: EMBRAPA-CPAC; 1998. p. 87-166.

SPAREMBERGUER, R. F. L.; SILVA, D. A. A relação homem, meio ambiente, desenvolvimento e o papel do direito ambiental. Veredas do Direito. 2005;2(4).

WOOLDRIDGE JM. Econometric analysis of cross section and panel data. Cambridge, Mass: MIT Press; 2002. 
XU L, LI B, YUAN Y, GAO X, ZHANG T, SUN Q. Detecting Different Types of Directional Land Cover Changes Using MODIS NDVI Time Series Dataset. Remote Sensing. 2016;8(6):495-518.

YUAN X, LI L, CHEN X, SHI H. Effects of Precipitation Intensity and Temperature on NDVI-Based Grass Change over Northern China during the Period from 1982 to 2011. Remote Sensing. 2015;7(8):10164-10183.

Sérgio Luís Dias Machado
Programa de Pós-Graduação em Qualidade Ambiental - Universidade Federal de Uberlândia, Minas
Gerais, Brasil.
E-mail: sergiodias.geo@gmail.com

Participação do autor: Contribuições substanciais para a concepção do trabalho, aquisição, análise e interpretação de dados, tratamento da imagens e geração do NDVI e redigiu o trabalho

Claudionor Ribeiro Silva

Programa de Pós-Graduação em Qualidade Ambiental - Universidade Federal de Uberlândia, Minas Gerais, Brasil

E-mail: crs.educ@gmail.com

Participação do autor: Contribuições substanciais para a concepção do trabalho, processamento, análise e interpretação das séries temporais, realizou uma revisão substancial

Aracy Alves de Araújo
Programa de Pós-Graduação em Qualidade Ambiental - Universidade Federal de Uberlândia, Minas
Gerais, Brasil.
E-mail: aracy@ufu.br

Participação do autor: Contribuições substanciais para a concepção do trabalho, processamento, análise e interpretação das séries temporais, realizou uma revisão substancial 